\title{
Brain Connectivity as Potential Biomarker for Alzheimer's Disease
}

\author{
Siddhartha Mondragón-Rodríguez 1,2,3 \\ ${ }^{1}$ CONACYT National Council for Science and Technology, México, México \\ ${ }^{2}$ UNAM Developmental Neurobiology and Neurophysiology, Institute of Neurobiology, National Autonomous University of México, Querétaro, \\ México \\ ${ }^{3}$ Douglas Hospital Research Center, Department of Psychiatry, McGill University, Montreal, Quebec, Canada
}

Corresponding author: Siddhartha Mondragón-Rodríguez, Ph.D., UNAM Developmental Neurobiology and Neurophysiology, Institute of Neurobiology, National Autonomous University of México, Boulevard Juriquilla 3001, Juriquilla, 76230 Santiago de Querétaro, Qro, México, Tel: (442) 238 1057; E-mail: sidmonrod@gmail.com

Received: Jun 21, 2016; Accepted: Jul 26, 2016; Published: Jul 29, 2016

Citation: Rodríguez SM. Brain Connectivity as Potential Biomarker for Alzheimer's Disease. J Neurol Neurosci. 2016, 7: S3.

\section{Abstract}

One important challenge for the Alzheimer's disease research field is developing new and efficacious biomarkers. In this regard we have devoted significant efforts towards finding biomarkers that are able to detect the prodromal pathological stage at an early phase. The idea behind this picture is to detect early stage points in order to provide better treatment options. Here, we firmly believe that this strategy will offer better hope for patients. With this in mind, we discuss the use of brain circuit alterations as a potential tool for early time point detection. Additionally, we briefly discuss the combination of powerful new techniques, like optogenetics and magnetic resonance imaging, for new diagnosis and treatment strategies.

The short document will be an important contribution towards exploring new strategies for $A D$ diagnosis and treatment. Given the practical application of these data, we feel that this manuscript will be of significant interest to the audience of Journal of Neurology and Neuroscience.

Keywords: Alzheimer's disease; Brain connectivity; Amyloid beta; Biomarker

\section{Introduction}

Alzheimer's disease (AD) has being canonically defined by the aberrant accumulation of amyloid beta $(A \beta)$ and tau protein aggregates [1]. The leading hypothesis postulates that accumulation in specific parts of the brain, i.e., hippocampus, causes neuronal death, translating into cognitive deficits [2]. It should be noted that the hippocampus is the paramount critical structure responsible for cognitive processing and memory formation [3]. The hypothesis could explain from a chronological perspective the pathogenesis and clinical signature of $A D$ patients; however, one logical corollary is missing from the clinical manifestation. Aspects of the symptomatology of $A D$ patients are not well correlated with protein deposition and the clinical $A D$ diagnosis is not well correlated with aggregates, particularly $A \beta$ deposition [4]. Indeed, several recent papers demonstrate the underling clinical pathology is not fundamentally underscored by protein deposition; rather, certain protein fragments are generated long before the main aggregate, the $A \beta$ plaque [5]. Currently, the soluble $A \beta$ version oligomers that are formed during the early phase of the amyloidogenesis are now being blamed for the neurotoxicity [6]. Although, several pieces of evidence have correlated the oligomeric version of $A \beta$ with synaptic plasticity and oscillatory network alterations, the precise relationship remains elusive [7]. For example, the newest peptide that is currently gaining attention among our research community is the b-CTF fragment [8]. This fragment has been correlated with alterations in oscillatory activity, which is critical for cognitive functions [8]. Alterations in two of the most important oscillatory rhythms, theta and gamma and their cross-frequency coupling, were found correlated with the b-CTF presence at one month of age in a transgenic mouse model that is characterized by developing $A \beta$ deposits at 4 months of age $[8,9]$. Further supporting these findings, we have found alterations in hippocampal theta activity in a different transgenic $A D$ mouse model which is characterized by $A \beta$ deposits at 6 months of age (unpublished data). In agreement to published data [8], our data suggests that even in less aggressive forms of $A D$ development, brain circuit activity is affected and consequently could be a putative critical marker during early stages of disease development.

The other eminent component in the proposed pathogenesis of $A D$ development has been tau deposits, mainly comprised of abnormally phosphorylated tau protein [1]. Abnormally phosphorylated tau protein aggregates within a neuron prompting to neuronal malfunction and finally to neuronal cell death, collaborating the tau hypothesis [4]. However, mouse models of tauopathy are absent for markers of cell death at early time stages, although aberrant hippocampal network functioning has been found [10]. More recent data from our group has demonstrated that brain network alterations are happening as early as one month of 
age in transgenic mouse models that are characterized by tau deposition at 8 months of age (unpublished data). Our data, along with published data [8], suggests that way before any potential tau deposits; brain circuit activity is drastically affected, thus supporting the idea of detecting malfunction in brain circuit activity as an early marker of disease progression [9]. This observation bridges the discussion for one of the current and therapeutic debates in the AD field, which is the treatment window for therapeutic intervention $[10,11]$. Here many authors, including us, believe that early intervention will offer better hope with better prognostic outcomes. Unfortunately, finding the early prodromal pathology of $A D$ has become particularly difficult; the consummate goal is to find early markers for disease progression. In this regard, we have seen that specific phosphorylation levels in tau protein could be observed as an early marker; nonetheless, the accuracy still depends mainly on the disease stage [12]. Concomitantly, brain circuit alterations, as seen in the transgenic models, are emerging as a remarkably reliable tool for early $A D$ diagnosis. The pending challenge now would be to translate these tools to human diagnosis. Nonetheless, less refined techniques currently used for brain circuit measurements like magnetic resonance imaging (MRI) are showing promising results [13]. In addition, new techniques like optogenetics, are helping us to dissect and understand brain circuit activity. The combination of powerful techniques such as optogenetics, MRI and electrophysiology, will become the key when designing protocols for diagnosis and treatment In this regard, functional MRI studies in patients with mild cognition impairment (prodromal stage of $A D$ ) have shown intranetwork functional disruptions within the dorsal attention network and executive control network [14]. Another functional MIR study showed similar results concluding that longitudinal alterations of functional connectivity are more profound in earlier stages as opposed to later stages of the disease [15]. Overall, is evident that brain circuit alterations are gaining attention as the new biomarker for the prodromal stage of AD.

The last three decades of $A D$ research has mainly focused on $A \beta$, which has been the main target for diagnosis and therapeutic approaches, but has shown little recuperative benefit [11]. In the last decade, a tau protein strategy has slowly become the newest $A \beta$ target, for both, diagnosis and therapeutic treatment. A fundamental question must be raised: are we willing to spend time and effort in developing a new potential protein treatment that is probably the wrong target? For the new generation of scientists, we have to derive new and fresh approaches; herein, brain circuit alterations as a new option for early diagnosis could offer better hope for $A D$ patients. Additionally, neural circuit alterations can become an interesting and more promising therapeutic strategy. In this vain, neural circuit stimulation has shown ameliorated effects in brain pathologies like Parkinson's disease [16]. With this in mind and the correct stimulation protocols, brain circuit strategy could become a promising therapeutic target for AD patients.

\section{Financial and Competing Interest's Disclosure}

The authors have no other relevant affiliations or financial involvement with any organization or entity with a financial interest in or financial conflict with the subject matter or materials discussed in the manuscript.

\section{Acknowledgment}

The authors thank Francis Manno III for critical comments. Siddhartha Mondragón-Rodríguez was awarded a Cátedra support from CONACYT, Mexico.

\section{References}

1. Mondragon RS (2008) Cleavage and conformational changes of tau protein follow phosphorylation during Alzheimer's disease. Int J Exp Pathol 89: 81-90.

2. Lassmann H (1995) Cell death in Alzheimer's disease evaluated by DNA fragmentation in situ. Acta Neuropathol 89: 35-41.

3. Geva S (2016) Impairment on a self-ordered working memory task in patients with early-acquired hippocampal atrophy. Dev Cogn Neurosci 20: 12-22.

4. Mondragon RS (2010) Causes versus effects: the increasing complexities of Alzheimer's disease pathogenesis. Expert Rev Neurother 10: 683-691.

5. Kostylev MA (2015) Prion-protein-interacting amyloid-beta oligomers of high molecular weight are tightly correlated with memory impairment in multiple Alzheimer mouse models. J Biol Chem 290: 17415-17438.

6. Itoh SG, Okumura H (2016) Oligomer formation of amyloid-beta (29-42) from its monomers using the Hamiltonian replicapermutation molecular dynamics simulation. J Phys Chem B.

7. Salgado PK, Pena OF (2015) Cellular and network mechanisms underlying memory impairment induced by amyloid beta protein. Protein Pept Lett 22: 303-321.

8. Goutagny R (2013) Alterations in hippocampal network oscillations and theta-gamma coupling arise before $A \beta$ overproduction in a mouse model of Alzheimer's disease. Eur J Neurosci 37: 1896-1902.

9. Mondragon RS (2013) Phosphorylation of tau protein as the link between oxidative stress, mitochondrial dysfunction, and connectivity failure: implications for Alzheimer's disease. Oxid Med Cell Longev 2013: 940603.

10. Booth CA (2016) Altered intrinsic pyramidal neuron properties and pathway-specific synaptic dysfunction underlie aberrant hippocampal network function in a mouse model of tauopathy. J Neurosci 36: 350-363.

11. Mondragon RS (2012) Amyloid beta and tau proteins as therapeutic targets for alzheimer's disease treatment: rethinking the current strategy. Int J Alzheimers Dis 2012: 630182.

12. Mondragon RS (2014) Phosphorylation of tau protein at sites Ser (396-404) is one of the earliest events in Alzheimer's disease and Down syndrome. Neuropathol Appl Neurobiol 40: 121-135. 
13. Trombella S (2016) Brain imaging of Alzheimer' disease: state of the art and perspectives for clinicians. Rev Med Suisse 12 795-798.

14. Zhu H (2016) Changes of intranetwork and internetwork functional connectivity in Alzheimer's disease and mild cognitive impairment. J Neural Eng 13: 046008.
15. Zhan $Y(2016)$ Longitudinal study of impaired intra- and internetwork brain connectivity in subjects at high risk for Alzheimer's Disease. J Alzheimers Dis 52: 913-927.

16. Pienaar IS (2015) Deep-brain stimulation associates with improved microvascular integrity in the subthalamic nucleus in Parkinson's disease. Neurobiol Dis 74: 392-405. 